\title{
A new series of large sets of subspace designs over the binary field
}

\author{
Michael Kiermaier, Reinhard Laue, Alfred Wassermann \\ Mathematisches Institut, University of Bayreuth \\ D-95440 Bayreuth, Germany \\ \{michael.kiermaier, reinhard.laue, alfred.wassermann\}@uni-bayreuth.de
}

September 18, 2018

\begin{abstract}
In this article, we show the existence of large sets $\mathrm{LS}_{2}[3](2, k, v)$ for infinitely many values of $k$ and $v$. The exact condition is $v \geq 8$ and $0 \leq k \leq v$ such that for the remainders $\bar{v}$ and $\bar{k}$ of $v$ and $k$ modulo 6 we have $2 \leq \bar{v}<$ $\bar{k} \leq 5$.

The proof is constructive and consists of two parts. First, we give a computer construction for an $\operatorname{LS}_{2}[3](2,4,8)$, which is a partition of the set of all 4-dimensional subspaces of an 8-dimensional vector space over the binary field into three disjoint $2-(8,4,217)_{2}$ subspace designs. Together with the already known $\operatorname{LS}_{2}[3](2,3,8)$, the application of a recursion method based on a decomposition of the Graßmannian into joins yields a construction for the claimed large sets.
\end{abstract}

\section{Introduction}

Let $V$ be a vector space of dimension $v$ over a finite field $\operatorname{GF}(q)$. For simplicity, a subspace of $V$ of dimension $k$ will be called an $k$-subspace. A (simple) $t$ - $(v, k, \lambda)_{q}$ subspace design $\mathcal{D}=(V, \mathcal{B})$ consists of a set $\mathcal{B}$ of $k$-subspaces of $V$, called blocks, such that each $t$-subspace of $V$ lies in exactly $\lambda$ blocks. This notion is a vector space analog of combinatorial $t$ designs on finite sets. For that reason, subspace designs are also called q-analogs of designs. Further names found in the literature include designs over finite fields, designs in vector spaces and designs in the q-Johnson scheme.

While combinatorial $t$-designs and Steiner systems have been studied since the 1830s and have a rich literature [12], the notion of subspace designs has been introduced by Cameron [9, 10] and Delsarte [13] in the 1970s. 
The set of all $k$-subspaces of $V$ is always a design, called trivial design. In 1987, Thomas [26] constructed the first non-trivial subspace design for $t=2$. Since then, more subspace designs have been constructed, see [6, 17, 8, 16, 18, 22, 24, 25].

A partition of the trivial design into $N$ disjoint $t-(v, k, \lambda)_{q}$ designs is called large set and denoted by $\operatorname{LS}_{q}[N](t, k, v)$. The value $\lambda$ is omitted in the parameter notation of a large set as $\lambda=\left[\begin{array}{l}v-t \\ k-t\end{array}\right]_{q}$ is already determined by the other parameters.

For ordinary combinatorial $t$-designs, large sets with $t=1$ exist if and only if $k$ divides $v$ [3]. In the $q$-analog case, this question is wide open, as it includes the question for the existence of parallelisms in projective geometries: A large set of 1- $(v, k, 1)_{q}$ designs (which are called spreads) is known as a $(k-1)$-parallelism of the projective geometry $\operatorname{PG}(v-1, q)$. To our knowledge, the only known existence results are the following: If $v \geq 2$ is a power of 2, then all 1-parallelisms do exist [14, 4. Furthermore, for $q=2$ and $v$ even, all 1-parallelisms do exist [2, 27]. In [15, a 1-parallelism of $\mathrm{PG}(5,3)$ is given. The only known parallelism with $k>1$ is a 2-parallelism of $\operatorname{PG}(5,2)[23$.

For $t \geq 2$, only the following large sets of subspace designs are known: There are computational constructions of an $\operatorname{LS}_{3}[2](2,3,6)[5]$, an $\operatorname{LS}_{2}[3](2,3,8)[8]$ and an $\mathrm{LS}_{5}[2](2,3,6)$ [7]. In [7, a recursive construction method was developed, which can be seen as a $q$ analog of the theory in [1], surveyed in [17. The application of this method to the $\mathrm{LS}_{3}[2](2,3,6)$ and $\mathrm{LS}_{5}[2](2,3,6)$ gave an infinite series of large sets $\mathrm{LS}_{q}(2, k, v)$ with $q \in\{3,5\}, v \geq 6, v \equiv 2(\bmod 4)$, and $3 \leq k \leq v-3, k \equiv 3(\bmod 4)$.

In this paper, we give a computational construction of an $\operatorname{LS}_{2}[3](2,4,8)$. The application of the recursion machinery of [7] to this large set and the already known $\mathrm{LS}_{2}[3](2,3,8)$ yields a new infinite series of large sets of subspace designs:

Theorem 1. Let $v$ and $k$ be integers with $v \geq 8$ and $0 \leq k \leq v$ such that

(i) $v \equiv 2 \bmod 6$ and $k \equiv 3,4,5 \bmod 6$ or

(ii) $v \equiv 3 \bmod 6$ and $k \equiv 4,5 \bmod 6$ or

(iii) $v \equiv 4 \bmod 6$ and $k \equiv 5 \bmod 6$.

Then there exists an $\mathrm{LS}_{2}[3](2, k, v)$.

Denoting the remainder of an integer $n$ modulo 6 by $\bar{n} \in\{0, \ldots, 5\}$, the conditions of Theorem 1(i), (ii) and (iii) can be stated as $2 \leq \bar{v}<\bar{k} \leq 5$.

\section{Preliminaries}

The set of all $k$-subspaces of $V$ is called the Graßmannian and is denoted by $\left[\begin{array}{l}V \\ k\end{array}\right]_{q}$. Our focus lies on the case $q=2$, where the 1-subspaces $\langle\mathbf{x}\rangle_{\mathrm{GF}(2)} \in\left[\begin{array}{l}V \\ 1\end{array}\right]_{2}$ are in one-to-one correspondence with the nonzero vectors $\mathbf{x} \in V \backslash\{\mathbf{0}\}$. The number of all $k$-subspaces of $V$ is given by the Gaussian binomial coefficient

$$
\#\left[\begin{array}{l}
V \\
k
\end{array}\right]_{q}=\left[\begin{array}{l}
v \\
k
\end{array}\right]_{q}= \begin{cases}\frac{\left(q^{v}-1\right) \cdots\left(q^{v-k+1}-1\right)}{\left(q^{k}-1\right) \cdots(q-1)} & \text { if } k \in\{0, \ldots, v\} \\
0 & \text { else. }\end{cases}
$$


The set $\mathcal{L}(V)$ of all subspaces of $V$ forms the subspace lattice of $V$.

By the fundamental theorem of projective geometry, for $v \neq 2$ the automorphism group of $\mathcal{L}(V)$ is given by the natural action of $\mathrm{P} \Gamma \mathrm{L}(V)$ on $\mathcal{L}(V)$. The automorphism group $\operatorname{Aut}(D)$ of a subspace design $D=(V, \mathcal{B})$ is defined as the stabilizer of $\mathcal{B}$ under this group action. Furthermore, for any subgroup $G \leq \operatorname{P\Gamma L}(V)$ we say that $D$ is $G$-invariant if $D^{G}=D$ or equivalently, $G \leq \operatorname{Aut}(D)$. In the case that $q$ is prime, the group $\operatorname{P\Gamma L}(V)$ reduces to $\operatorname{PGL}(V)$, and for the case of our interest $q=2$, it reduces further to $\mathrm{GL}(V)$. After a choice of a basis of $V$, its elements are represented by the invertible $v \times v$ matrices $A$, and the action on $\mathcal{L}(V)$ is given by the vector-matrix-multiplication $\mathbf{v} \mapsto \mathbf{v} A$.

As $t$-design, the trivial design $\left(V,\left[\begin{array}{l}V \\ k\end{array}\right]_{q}\right)$ has parameters $t$ - $\left(v, k, \lambda_{\max }\right)_{q}$, where

$$
\lambda_{\max }=\left[\begin{array}{l}
v-t \\
k-t
\end{array}\right]_{q} .
$$

Hence, an obvious necessary condition for the existence of an $\operatorname{LS}_{q}[N](t, k, v)$ is the equality $\lambda \cdot N=\lambda_{\max }$. Moreover, since the blocks of a $t$-design also form an $i$-design for $i \in\{0, \ldots, t\}$, we have the necessary conditions

$$
N \mid\left[\begin{array}{l}
v-i \\
k-i
\end{array}\right]_{q} \text { for } i \in\{0, \ldots, t\}
$$

A parameter set $\operatorname{LS}_{q}[N](t, k, v)$ is called admissible if all the necessary conditions are fulfilled. If moreover an $\operatorname{LS}_{q}[N](t, k, v)$ actually exists, the parameter set is called realizable. In the following, it proves useful to extend the parameters to the value $t=-1$ by unconditionally accepting all the large set parameters of the form $\operatorname{LS}_{q}[N](-1, k, v)$ as admissible and realizable.

By [18, Cor. 19], the existence of some $\operatorname{LS}_{q}[N](t, k, v)$ with $t \geq 1$ implies the existence of derived large sets $\operatorname{LS}_{q}[N](t-1, k-1, v-1)$, residual large sets $\operatorname{LS}_{q}[N](t-1, k, v-1)$ and the dual large set $\operatorname{LS}_{q}[N](t, v-k, v)$. Furthermore, the existence of $\operatorname{LS}_{q}[N](t, k-1, v-1)$ and $\operatorname{LS}_{q}[N](t, k, v-1)$ implies the existence of an $\operatorname{LS}_{q}[N](t, k, v)$ [18, Cor. 20].

The following theory is needed for the recursive construction of large sets. For more details and proofs, see [7].

Two subsets $\mathcal{B}_{1}$ and $\mathcal{B}_{2}$ of $\left[\begin{array}{l}V \\ k\end{array}\right]_{q}$ are called $t$-equivalent if

$$
\#\left\{B \in \mathcal{B}_{1} \mid T \leq B\right\}=\#\left\{B \in \mathcal{B}_{2} \mid T \leq B\right\}
$$

for all $t$-subspaces $T$ of $V$. In this situation, the pair $\left(\mathcal{B}_{1}, \mathcal{B}_{2}\right)$ has also been called trade or bitrade, see for example [20, 21] for some recent results. Furthermore, given integers $0 \leq t \leq k \leq v, N \geq 2$ and a set $\mathcal{B}$ of $k$-subspaces of $V$, a partition $\left\{\mathcal{B}_{1}, \ldots, \mathcal{B}_{N}\right\}$ of $\mathcal{B}$ is called an $(N, t)$-partition, if the parts $\mathcal{B}_{i}$ are pairwise $t$-equivalent. The notion of $(N, t)$-partitions can be seen as a generalization of large sets, as by [7, Lemma 4.8], $\left(V,\left\{\mathcal{B}_{1}, \ldots, \mathcal{B}_{N}\right\}\right)$ is an $\operatorname{LS}_{q}[N](t, k, v)$ if and only if $\left\{\mathcal{B}_{1}, \ldots, \mathcal{B}_{N}\right\}$ is an $(N, t)$-partition of $\left[\begin{array}{l}V \\ k\end{array}\right]_{q}$.

A set $\mathcal{B}$ of $k$-subspaces is called $(N, t)$-partitionable if there exists an $(N, t)$-partition of $\mathcal{B}$. Again, the notion is extended to $t=-1$ by unconditionally calling any set 
of $k$-subspaces of $V(N,-1)$-partitionable. We have the following structure properties: If $\mathcal{B}$ is $(N, t)$-partitionable, it is $(N, s)$-partitionable for all $s \in\{-1, \ldots, t\}[7$, Lemma 4.3]. The disjoint union of $(N, t)$-partitionable sets is again $(N, t)$-partitionable [7, Lemma 4.7]. Therefore, we can construct an $\operatorname{LS}_{q}[N](t, k, v)$ by decomposing $\left[\begin{array}{l}V \\ k\end{array}\right]_{q}$ into $(N, t)$-partitionable sets.

The known constructions of large sets of classical combinatorial designs and subspace designs often rely on a decomposition into so-called joins. In [7, three kinds of joins are provided. For our purpose, we only need one of them: For a chain of subspaces $K_{1} \leq U_{1} \leq U_{2} \leq K_{2} \leq V$, the avoiding join of $K_{1}$ and $K_{2}$ with respect to the factor space $F=U_{2} / U_{1}$ is given by

$$
K_{1} *_{\bar{F}} K_{2} / U_{2}=\left\{K \in \mathcal{L}(V) \mid U_{1} \cap K=K_{1}, U_{2}+K=K_{2}, U_{1} \cap K=U_{2} \cap K\right\} .
$$

By [7, Lemma 3.7], $K_{1} *_{\bar{F}} K_{2} / U_{2}$ consists of $q^{\left(\operatorname{dim}\left(U_{1}\right)-\operatorname{dim}\left(K_{1}\right)\right)\left(\operatorname{dim}\left(K_{2}\right)-\operatorname{dim}\left(U_{1}\right)\right)}$ subspaces of $V$ of dimension $k_{1}+k_{2}-u_{1}$. The definition is extended to sets $\mathcal{B}^{(1)} \subseteq\left[\begin{array}{l}U_{1} \\ k_{1}\end{array}\right]_{q}$ and $\mathcal{B}^{(2)} \subseteq\left[\begin{array}{c}V / U_{2} \\ \bar{k}_{2}\end{array}\right]_{q}$ of subspaces by setting

$$
\mathcal{B}^{(1)} * \mathcal{B}^{(2)}=\bigcup_{\substack{B^{(1)} \in \mathcal{B}^{(1)} \\ B^{(2)} \in \mathcal{B}^{(2)}}} B^{(1)} * B^{(2)} .
$$

The power of the join for the construction of $(N, t)$-partitionable sets is rooted in the following lemma. In [7, Lemma 4.10] it is stated and proven for all three kinds of joins.

Lemma 2.1 (Basic Lemma for the avoiding join). Let $U_{1} \leq U_{2} \leq V$ be a chain of subspaces, $k_{1} \in\left\{0, \ldots, \operatorname{dim}\left(U_{1}\right)\right\}, \bar{k}_{2} \in\left\{0, \ldots, \operatorname{dim}\left(V / U_{2}\right)\right\}$ and $N$ a positive integer. If $\mathcal{B}^{(1)} \subseteq\left[\begin{array}{l}U_{1} \\ k_{1}\end{array}\right]_{q}$ is $\left(N, t_{1}\right)$-partitionable and $\mathcal{B}^{(2)} \subseteq\left[\begin{array}{c}V / U_{2} \\ \bar{k}_{2}\end{array}\right]_{q}$ is $\left(N, t_{2}\right)$-partitionable with integers $t_{1}, t_{2} \geq-1$, then the avoiding join $\mathcal{B}^{(1)} * \frac{U_{U_{2} / U_{1}}}{\mathcal{B}^{(2)}}$ is $\left(N, t_{1}+t_{2}+1\right)$-partitionable.

By [7, Theorem 3.19], for a maximal chain

$$
\{\mathbf{0}\}=U_{0}<U_{1}<\ldots<U_{v}=V
$$

of subspaces and $s \in\{0, \ldots, v-k-1\}$, a partition of $\left[\begin{array}{l}V \\ k\end{array}\right]_{q}$ into avoiding joins is given by the disjoint union

$$
\left[\begin{array}{l}
V \\
k
\end{array}\right]_{q}=\bigcup_{i=0}^{k}\left[\begin{array}{c}
U_{s+i} \\
i
\end{array}\right]_{q} * \overline{U_{s+i+1} / U_{s+i}}\left[\begin{array}{c}
V / U_{s+i+1} \\
k-i
\end{array}\right]_{q} .
$$

For the construction of large sets, only the dimensions of the involved Graßmannians are relevant. Reducing the notation to this information, we say that the above decomposition has the decomposition type

$$
\left[\begin{array}{l}
v \\
k
\end{array}\right]=\bigcup_{i=0}^{k}\left[\begin{array}{c}
s+i \\
i
\end{array}\right] *\left[\begin{array}{c}
v-s-i-1 \\
k-i
\end{array}\right] .
$$

By the above discussion, the application of the Basic Lemma 2.1 to this decomposition yields 
Proposition 2.2. Let $0 \leq t \leq k \leq v, N \geq 2$ and $s \in\{0, \ldots, v-k-1\}$ be integers. If for each $i \in\{0, \ldots, k\}$ there are integers $t_{1}, t_{2} \geq-1$ with $t_{1}+t_{2}+1 \geq t$ such that $\mathrm{LS}_{q}[N]\left(t_{1}, i, s+i\right)$ and $\mathrm{LS}_{q}[N]\left(t_{2}, k-i, v-s-i-1\right)$ both are realizable, then $\mathrm{LS}_{q}[N](t, k, v)$ is realizable.

We remark that the proof of the Basic Lemma in [7] is constructive, implying that the statement of Proposition 2.2 is constructive, too.

\section{The method of Kramer and Mesner}

Fixing a subgroup $G$ of $\mathrm{P} \Gamma \mathrm{L}(V)$, the following idea can be used for the construction of $G$ invariant $t-(v, k, \lambda)_{q}$ designs [19, 22, 6]: The action of $G$ induces partitions $\left[\begin{array}{c}V \\ t\end{array}\right]_{q}=\bigcup_{i=1}^{\tau} \mathcal{T}_{i}$ and $\left[\begin{array}{l}V \\ k\end{array}\right]_{q}=\bigcup_{j=1}^{\kappa} \mathcal{K}_{j}$ into orbits. Any $G$-invariant subspace design will have the form $(V, \mathcal{B})$ with $\mathcal{B}=\bigcup_{j \in J} \mathcal{K}_{j}$ and $J \subseteq\{1, \ldots, \kappa\}$. In this way, an index set $J \subseteq\{1, \ldots, \kappa\}$ gives a $t-(v, k, \lambda)_{q}$ design if and only if its characteristic vector $\chi_{J} \in\{0,1\}^{\kappa}$ is a solution of the system of linear integer equations

$$
A_{t, k}^{G} \chi_{J}=\lambda \mathbf{1}
$$

where $\mathbf{1}$ is the all-one vector and $A_{t, k}^{G}=\left(a_{i j}\right)$ is the $(\kappa \times \tau)$-matrix with the entries

$$
a_{i j}=\#\left\{K \in \mathcal{K}_{j} \mid T_{i} \leq K\right\} .
$$

Here, $T_{i} \in \mathcal{T}_{i}$ denotes a set of orbit representatives. The matrix $A_{t, k}^{G}$ is called $G$-incidence matrix.

For the construction of large sets, we iterate this method as in [8]: After finding a solution $\chi_{J}$, we remove all the columns from $A_{t, k}^{G}$ having an index $j \in J$. Now the solutions of the reduced system give precisely the $G$-invariant subspace designs which are disjoint to $D$. If we succeed in repeating the process until all $G$-orbits $\mathcal{K}_{j}$ are covered, we have constructed a large set consisting of $t-(v, k, \lambda)_{q}$ designs. The same approach has been used by Chee et al [1] to construct large sets of designs over sets.

\section{An $\mathrm{LS}_{2}[3](2,4,8)$}

A $\mathrm{LS}_{2}[3](2,4,8)$ consists of three mutually disjoint $2-(8,4,217)_{2}$ designs. It is worth noting that up to now, even the existence of a single design with these parameters was open.

We succeeded in the construction of an $\operatorname{LS}_{2}[3](2,4,8)$ by the Kramer-Mesner method described above. For that purpose, we take the 8-dimensional $\mathrm{GF}(2)$ vector space $V=$ $\mathrm{GF}\left(2^{8}\right)$ and prescribe the group $G=\left\langle\sigma^{5}, \phi^{2}\right\rangle$ of order 204, where $\sigma: x \mapsto x \alpha$ with a primitive element $\alpha$ of $\mathrm{GF}\left(2^{8}\right)$ is a Singer cycle and $\phi: x \mapsto x^{2}$ is the Frobenius automorphism. After the choice of a suitable basis of $V$, the generators can be written 


$$
\sigma^{5}=\left(\begin{array}{cccccccc}
0 & 0 & 0 & 0 & 0 & 1 & 0 & 0 \\
0 & 0 & 0 & 0 & 0 & 0 & 1 & 0 \\
0 & 0 & 0 & 0 & 0 & 0 & 0 & 1 \\
1 & 0 & 1 & 1 & 1 & 0 & 0 & 0 \\
0 & 1 & 0 & 1 & 1 & 1 & 0 & 0 \\
0 & 0 & 1 & 0 & 1 & 1 & 1 & 0 \\
0 & 0 & 0 & 1 & 0 & 1 & 1 & 1 \\
1 & 0 & 1 & 1 & 0 & 0 & 1 & 1
\end{array}\right) \quad \text { and } \quad \phi^{2}=\left(\begin{array}{cccccccc}
1 & 0 & 0 & 0 & 0 & 0 & 0 & 0 \\
0 & 0 & 0 & 0 & 1 & 0 & 0 & 0 \\
1 & 0 & 1 & 1 & 1 & 0 & 0 & 0 \\
1 & 0 & 1 & 1 & 0 & 0 & 1 & 1 \\
0 & 0 & 1 & 1 & 0 & 0 & 1 & 0 \\
0 & 0 & 1 & 0 & 1 & 1 & 0 & 1 \\
1 & 1 & 1 & 1 & 0 & 0 & 0 & 1 \\
0 & 0 & 0 & 1 & 1 & 0 & 0 & 0
\end{array}\right)
$$

In Tables 1, 2, and 3 we list the orbit representatives for the three mutually disjoint designs $\mathcal{D}_{1}, \mathcal{D}_{2}$, and $\mathcal{D}_{3}$ we found, using the same encoding as in [8]. For each representative, the four row vectors

$$
\left[\begin{array}{llll}
w_{0} & w_{1} & \ldots & w_{7} \\
x_{0} & x_{1} & \ldots & x_{7} \\
y_{0} & y_{1} & \ldots & y_{7} \\
z_{0} & z_{1} & \ldots & z_{7}
\end{array}\right]
$$

spanning a 3 -subspace of $V$, are encoded as a quadruple of positive integers

$$
[W, X, Y, Z]=\left[\sum_{i=0}^{7} w_{i} 2^{i}, \sum_{i=0}^{7} x_{i} 2^{i}, \sum_{i=0}^{7} y_{i} 2^{i}, \sum_{i=0}^{7} z_{i} 2^{i}\right] .
$$

\section{An infinite series of large sets}

Now we are ready to proof our main result.

Proof of Theorem 1. We proceed by induction on $v$. For $v=8$, an $\operatorname{LS}_{2}[3](2,4,8)$ was constructed above. In $\left[8\right.$, an $\operatorname{LS}_{2}[3](2,3,8)$ was constructed, and its dual large set is an $\operatorname{LS}_{2}[3](2,5,8)$. The repeated application of [18, Cor. 20] yields the existence of $\mathrm{LS}_{2}[3](2,4,9), \mathrm{LS}_{2}[3](2,5,9)$ and $\mathrm{LS}_{2}[3](2,5,10)$.

It remains to consider $v \geq 14$. By duality, we may assume $k \leq \frac{v}{2}$. By $v-k-1 \geq$ $\frac{v}{2}-1 \geq 6$ and the realizability statements of Table 4 , the existence of an $\operatorname{LS}_{2}[3](2, k, v)$ follows from the application of Proposition 2.2 with $s=5$.

It remains to show the correctness of Table 4, For all $i \equiv 3,4,5 \bmod 6$, there exists an $\operatorname{LS}_{2}[3](2, i, 5+i)$ by the induction hypothesis. By taking a derived large set, we see that for $i \equiv 2 \bmod 6$, there exists an $\mathrm{LS}_{2}[3](1, i, 5+i)$ and for $i \equiv 1 \bmod 6$, there exists an $\operatorname{LS}_{2}[3](0, i, 5+i)$. Similarly, for $i \equiv 0 \bmod 6$, we have an $\operatorname{LS}_{2}[3](2, k-i, v-6-i)$ by the induction hypothesis. By derived large sets, for $i \equiv 5 \bmod 6$ there exists an $\mathrm{LS}_{2}[3](1, k-i, v-6-i)$, and for $i \equiv 4 \bmod 6$ there exists an $\mathrm{LS}_{2}[3](0, k-i, v-6-i)$. 
Table 1: $2-(8,4,217 ; 2)$ design $\mathcal{B}_{1}$

\begin{tabular}{|c|c|c|}
\hline$[1,34,40,192]$ & {$[33,34,132,112]$} & {$[67,136,16,32]$} \\
\hline$[1,34,84,128]$ & {$[33,34,168,192]$} & {$[67,196,40,48]$} \\
\hline$[1,34,104,16]$ & {$[33,34,196,184]$} & {$[69,74,16,224]$} \\
\hline$[1,66,244,40]$ & {$[33,66,28,128]$} & {$[69,142,80,96]$} \\
\hline$[1,98,172,16]$ & {$[33,98,36,48]$} & {$[69,210,136,96]$} \\
\hline$[1,106,60,128]$ & {$[33,98,36,176]$} & {$[73,66,204,144]$} \\
\hline$[1,106,76,16]$ & {$[33,130,36,40]$} & {$[73,74,84,32]$} \\
\hline$[1,106,236,240]$ & {$[33,136,16,64]$} & {$[73,76,208,224]$} \\
\hline$[1,114,116,72]$ & {$[33,148,168,192]$} & {$[73,162,44,176]$} \\
\hline$[1,130,88,32]$ & {$[33,170,108,48]$} & {$[73,202,196,96]$} \\
\hline$[1,134,72,176]$ & {$[33,178,52,232]$} & {$[73,226,76,176]$} \\
\hline$[1,134,104,80]$ & {$[33,210,100,200]$} & {$[81,34,108,128]$} \\
\hline$[1,162,4,248]$ & {$[34,164,72,208]$} & {$[81,90,28,96]$} \\
\hline$[1,170,44,112]$ & {$[35,132,144,192]$} & {$[81,98,244,136]$} \\
\hline$[1,170,76,208]$ & {$[37,38,40,80]$} & {$[81,130,164,88]$} \\
\hline$[1,194,20,88]$ & {$[37,86,72,128]$} & {$[81,146,200,224]$} \\
\hline$[1,210,68,88]$ & {$[41,10,36,144]$} & {$[81,210,180,120]$} \\
\hline$[1,210,148,200]$ & {$[41,34,28,64]$} & {$[82,116,8,128]$} \\
\hline$[1,214,136,160]$ & {$[41,74,108,144]$} & {$[85,194,216,224]$} \\
\hline$[1,226,104,144]$ & {$[41,98,28,128]$} & {$[85,198,8,224]$} \\
\hline$[1,226,148,56]$ & {$[41,234,12,208]$} & {$[89,42,124,128]$} \\
\hline$[1,228,168,240]$ & {$[49,2,132,200]$} & {$[89,74,12,32]$} \\
\hline$[1,242,84,136]$ & {$[49,6,40,64]$} & {$[97,2,100,80]$} \\
\hline$[2,4,8,112]$ & {$[49,18,4,104]$} & {$[97,34,4,248]$} \\
\hline$[2,4,88,128]$ & {$[49,34,4,184]$} & {$[97,34,12,208]$} \\
\hline$[2,4,168,112]$ & {$[49,34,100,40]$} & {$[97,34,20,248]$} \\
\hline$[2,8,16,224]$ & {$[49,82,4,248]$} & {$[97,36,232,176]$} \\
\hline$[2,36,104,80]$ & {$[49,114,36,152]$} & {$[97,42,196,144]$} \\
\hline$[3,44,176,192]$ & {$[49,114,116,216]$} & {$[97,50,68,168]$} \\
\hline$[5,106,16,128]$ & {$[49,130,36,72]$} & {$[97,50,228,168]$} \\
\hline$[9,18,20,160]$ & {$[49,130,116,200]$} & {$[97,66,44,80]$} \\
\hline$[9,66,44,176]$ & {$[53,146,24,192]$} & {$[97,66,52,24]$} \\
\hline$[9,74,212,96]$ & {$[57,98,108,128]$} & {$[97,100,200,240]$} \\
\hline$[9,82,204,32]$ & {$[57,186,28,64]$} & {$[97,106,204,240]$} \\
\hline$[9,170,140,64]$ & {$[65,4,16,128]$} & {$[97,130,132,136]$} \\
\hline$[10,68,80,96]$ & {$[65,10,108,112]$} & {$[97,130,168,80]$} \\
\hline$[13,10,144,64]$ & {$[65,34,104,112]$} & {$[97,194,84,104]$} \\
\hline$[13,70,208,160]$ & {$[65,82,132,168]$} & {$[97,194,148,136]$} \\
\hline$[13,202,16,160]$ & {$[65,82,212,216]$} & {$[97,194,200,144]$} \\
\hline$[17,18,20,136]$ & {$[65,82,228,104]$} & {$[97,226,132,144]$} \\
\hline$[17,18,164,40]$ & {$[65,130,8,32]$} & {$[97,228,136,16]$} \\
\hline$[17,24,64,128]$ & {$[65,130,24,160]$} & {$[98,164,72,208]$} \\
\hline$[17,34,4,104]$ & {$[65,130,180,56]$} & {$[98,228,8,112]$} \\
\hline$[17,50,20,152]$ & {$[65,130,212,72]$} & {$[101,134,232,16]$} \\
\hline$[17,66,4,200]$ & {$[65,146,212,248]$} & {$[105,12,48,128]$} \\
\hline$[17,82,200,160]$ & {$[65,162,100,16]$} & {$[105,74,196,176]$} \\
\hline$[17,130,148,24]$ & {$[65,170,108,176]$} & {$[105,162,196,240$} \\
\hline$[17,130,228,56]$ & {$[65,194,36,216]$} & {$[113,18,196,232]$} \\
\hline$[17,146,20,136]$ & {$[65,194,52,8]$} & {$[113,34,116,232]$} \\
\hline$[17,146,132,152]$ & {$[65,194,196,168]$} & {$[113,66,212,120]$} \\
\hline$[17,218,20,96]$ & {$[65,202,92,160]$} & {$[113,82,180,72]$} \\
\hline$[17,242,228,104]$ & {$[66,68,8,128]$} & {$[113,114,116,232$} \\
\hline$[18,116,104,128]$ & {$[66,68,72,224]$} & {$[113,130,4,136]$} \\
\hline$[19,36,104,128]$ & {$[66,140,16,160]$} & {$[113,130,180,248$} \\
\hline$[21,146,136,160]$ & {$[67,4,8,208]$} & {$[113,194,84,248]$} \\
\hline$[21,150,24,96]$ & {$[67,4,56,128]$} & {$[113,226,212,232$} \\
\hline$[25,68,32,128]$ & {$[67,36,136,208]$} & {$[113,242,244,200$} \\
\hline$[33,18,100,184]$ & {$[67,68,136,224]$} & {$[115,36,72,128]$} \\
\hline
\end{tabular}

$\begin{array}{ll}{[129,10,4,16]} & {[145,154,196,160]} \\ {[129,34,68,40]} & {[145,178,68,248]} \\ {[129,66,180,216]} & {[145,226,164,232]} \\ {[129,82,116,88]} & {[147,68,72,32]} \\ {[129,98,36,168]} & {[147,132,88,224]} \\ {[129,98,40,16]} & {[149,86,152,224]} \\ {[129,98,44,208]} & {[149,214,200,32]} \\ {[129,100,168,80]} & {[153,82,68,96]} \\ {[129,114,52,152]} & {[153,130,4,224]} \\ {[129,114,52,200]} & {[153,146,132,160]} \\ {[129,114,100,8]} & {[161,26,52,64]} \\ {[129,114,116,232]} & {[161,34,84,168]} \\ {[129,130,8,64]} & {[161,34,108,240]} \\ {[129,130,52,184]} & {[161,34,196,112]} \\ {[129,162,132,88]} & {[161,42,68,240]} \\ {[129,194,84,184]} & {[161,66,68,80]} \\ {[129,194,204,208]} & {[161,74,36,16]} \\ {[129,210,164,56]} & {[161,74,76,208]} \\ {[129,210,216,32]} & {[161,82,4,120]} \\ {[129,226,40,144]} & {[161,98,228,16]} \\ {[129,226,228,40]} & {[161,114,228,168]} \\ {[129,242,244,168]} & {[161,138,228,16]} \\ {[130,4,40,192]} & {[161,146,164,136]} \\ {[130,12,16,32]} & {[161,162,20,200]} \\ {[130,20,8,96]} & {[161,170,176,192]} \\ {[130,164,200,80]} & {[161,178,44,192]} \\ {[131,4,136,240]} & {[161,226,196,40]} \\ {[131,132,72,32]} & {[161,234,172,112]} \\ {[133,34,72,240]} & {[161,242,52,104]} \\ {[133,166,40,176]} & {[162,40,48,64]} \\ {[133,166,200,208]} & {[163,132,200,16]} \\ {[133,202,16,96]} & {[163,228,168,176]} \\ {[137,2,20,224]} & {[163,228,232,48]} \\ {[137,76,16,160]} & {[165,146,152,192]} \\ {[137,82,68,160]} & {[169,10,12,112]} \\ {[137,90,204,32]} & {[169,34,100,144]} \\ {[137,106,44,176]} & {[169,98,164,240]} \\ {[137,130,196,96]} & {[169,130,236,176]} \\ {[137,138,108,208]} & {[169,170,44,176]} \\ {[137,154,76,160]} & {[173,138,176,64]} \\ {[137,158,32,192]} & {[177,2,84,248]} \\ {[137,170,100,80]} & {[177,34,244,248]} \\ {[137,172,176,192]} & {[177,42,164,64]} \\ {[137,194,236,176]} & {[177,66,52,120]} \\ {[138,132,176,64]} & {[177,66,84,136]} \\ {[138,156,160,192]} & {[177,114,116,72]} \\ {[141,14,48,64]} & {[177,150,56,192]} \\ {[145,2,20,184]} & {[177,162,20,8]} \\ {[145,2,92,32]} & {[177,194,148,184]} \\ {[145,34,60,64]} & {[177,210,100,248]} \\ {[145,66,132,152]} & {[177,210,116,72]} \\ {[145,82,164,136]} & {[177,226,52,40]} \\ {[145,98,36,88]} & {[177,226,84,120]} \\ {[145,114,132,72]} & {[185,178,44,64]} \\ {[145,114,164,152]} & {[193,2,8,160]} \\ {[145,130,52,232]} & {[193,10,228,48]} \\ {[145,138,20,64]} & {[193,34,204,208]} \\ {[145,146,92,32]} & {[193,68,232,112]} \\ & \end{array}$

$[193,98,4,40]$ $193,178,4,8]$ $[193,178,20,168$ $[193,196,136,240]$ $[193,226,72,48]$ $[193,226,84,40]$ $[193,226,84,40]$ $[194,36,232,112]$ $[194,36,232,112]$ $[194,68,136,112]$
$[197,102,168,144]$ $[197,102,168,144]$ $[197,150,8,32]$ $[197,194,200,208]$ $197,214,152,224]$ $[198,8,16,160]$ $[201,82,28,32]$ $[201,162,100,176]$ [209, 26, 212, 96] $[209,50,164,216]$ $[209,56,164,216]$ $[209,114,116,216]$ $[209,114,116,216]$ $[209,130,84,8]$ $[209,146,52,248]$ $[209,148,8,96]$ 209, 154, 68, 224] $[209,178,36,152$ $[209,178,68,136]$ [209, 212, 8, 96] $[209,242,164,24]$ $[217,26,68,224]$ $[217,194,68,96]$ $[217,210,204,160]$ $[225,10,12,48]$ $[225,38,136,112]$ $[225,98,164,48]$ $[225,170,132,144]$ $[225,170,204,112]$ $[225,210,100,40]$ $[225,230,136,144]$ $[226,36,232,144]$ $[227,36,136,80]$ $[227,132,168,144]$ $229,6,72,144$ $[233,130,4,16]$ $233,226,12,144$ $[233,226,68,80]$ $[241,2,180,40]$ $[241,50,84,136]$ $[241,50,244,216]$ $[241,66,132,136]$ $[241,82,148,136]$ $241,114,164,104$ $[241,162,4,248]$
$[241,242,180,56]$ 
Table 2: 2- $(8,4,217 ; 2)$ design $\mathcal{B}_{2}$

\begin{tabular}{|c|c|c|c|c|}
\hline$[1,2,80,32]$ & {$[17,226,164,104]$} & {$[65,82,220,32]$} & {$[101,134,200,240]$} & {$[145,226,52,40]$} \\
\hline$[1,2,140,160]$ & {$[17,242,68,248]$} & {$[65,102,136,80]$} & {$[105,10,28,128]$} & {$[145,242,196,216]$} \\
\hline$[1,4,232,240]$ & {$[18,4,24,96]$} & {$[65,106,204,16]$} & {$[105,106,100,48]$} & {$[149,6,160,192]$} \\
\hline$[1,6,104,48]$ & {$[18,148,72,96]$} & {$[65,114,52,216]$} & {$[105,130,140,240]$} & {$[149,18,32,192]$} \\
\hline$[1,6,136,144]$ & {$[19,84,136,224]$} & {$[65,130,136,240]$} & {$[105,226,172,208]$} & {$[149,194,88,224]$} \\
\hline$[1,10,204,112]$ & {$[21,134,56,64]$} & {$[65,146,196,224]$} & {$[105,234,68,112]$} & {$[149,214,200,160]$} \\
\hline$[1,42,112,128]$ & {$[23,72,32,128]$} & {$[65,162,116,168]$} & {$[113,50,84,168]$} & {$[153,82,212,224]$} \\
\hline$[1,42,164,240]$ & {$[25,58,124,128]$} & {$[65,162,164,176]$} & {$[113,50,108,128]$} & {$[153,194,140,160]$} \\
\hline$[1,66,100,176]$ & {$[25,66,148,32]$} & {$[65,170,196,176]$} & {$[113,50,196,8]$} & {$[161,2,100,176]$} \\
\hline$[1,66,244,8]$ & {$[25,90,20,128]$} & {$[65,196,152,160]$} & {$[113,54,104,128]$} & {$[161,2,164,88]$} \\
\hline$[1,82,148,200]$ & {$[25,90,92,160]$} & {$[65,198,104,80]$} & {$[113,162,228,248]$} & {$[161,18,100,248]$} \\
\hline$[1,90,12,128]$ & {$[25,154,148,96]$} & {$[66,20,88,32]$} & {$[113,226,100,104]$} & {$[161,42,16,192]$} \\
\hline$[1,98,40,128]$ & {$[25,194,20,224]$} & {$[66,212,88,96]$} & {$[113,242,100,184]$} & {$[161,50,132,56]$} \\
\hline$[1,132,40,48]$ & {$[25,210,196,224]$} & {$[67,4,208,96]$} & {$[115,36,8,128]$} & {$[161,66,40,176]$} \\
\hline$[1,134,104,240]$ & {$[29,74,32,128]$} & {$[67,68,136,96]$} & {$[129,2,52,192]$} & {$[161,82,36,8]$} \\
\hline$[1,146,20,224]$ & {$[33,6,40,128]$} & {$[67,196,104,80]$} & {$[129,34,72,240]$} & {$[161,130,136,176]$} \\
\hline$[1,146,196,168]$ & {$[33,6,104,176]$} & {$[67,228,72,208]$} & {$[129,38,200,176]$} & {$[161,130,228,72]$} \\
\hline$[1,162,100,48]$ & {$[33,18,20,72]$} & {$[69,82,24,32]$} & {$[129,50,100,88]$} & {$[161,138,76,112]$} \\
\hline$[1,170,172,240]$ & {$[33,90,84,128]$} & {$[69,130,24,32]$} & {$[129,50,148,184]$} & {$[161,138,164,144]$} \\
\hline$[1,182,56,64]$ & {$[33,98,68,208]$} & {$[73,34,228,48]$} & {$[129,66,4,8]$} & {$[161,164,232,176]$} \\
\hline$[1,194,40,176]$ & {$[33,130,100,72]$} & {$[73,66,68,16]$} & {$[129,66,12,80]$} & {$[161,194,36,56]$} \\
\hline$[1,194,76,240]$ & {$[33,130,148,88]$} & {$[73,138,36,48]$} & {$[129,74,156,96]$} & {$[161,210,148,72]$} \\
\hline$[1,196,136,176]$ & {$[33,130,212,88]$} & {$[73,170,4,208]$} & {$[129,74,204,224]$} & {$[162,132,8,48]$} \\
\hline$[1,202,196,224]$ & {$[33,146,116,136]$} & {$[73,194,76,160]$} & {$[129,100,136,208]$} & {$[162,164,136,112]$} \\
\hline$[1,210,132,24]$ & {$[33,162,56,192]$} & {$[73,218,68,224]$} & {$[129,130,72,96]$} & {$[162,180,184,192]$} \\
\hline$[1,210,196,24]$ & {$[33,162,180,24]$} & {$[73,226,68,144]$} & {$[129,130,212,120]$} & {$[163,36,48,64]$} \\
\hline$[1,226,164,232]$ & {$[33,178,244,200]$} & {$[81,18,20,56]$} & {$[129,134,136,240]$} & {$[163,196,104,80]$} \\
\hline$[1,230,232,80]$ & {$[33,194,136,80]$} & {$[81,54,104,128]$} & {$[129,146,20,40]$} & {$[165,50,40,192]$} \\
\hline$[2,4,184,64]$ & {$[33,210,84,72]$} & {$[81,74,84,128]$} & {$[129,162,40,80]$} & {$[169,130,12,16]$} \\
\hline$[2,84,88,160]$ & {$[33,226,4,56]$} & {$[81,82,132,216]$} & {$[129,210,88,96]$} & {$[169,138,156,64]$} \\
\hline$[3,68,8,224]$ & {$[35,132,72,112]$} & {$[81,98,164,200]$} & {$[129,226,44,144]$} & {$[169,186,148,64]$} \\
\hline$[3,68,232,144]$ & {$[35,132,136,112]$} & {$[81,130,132,40]$} & {$[130,36,200,80]$} & {$[169,194,164,112]$} \\
\hline$[3,132,168,176]$ & {$[35,164,8,208]$} & {$[81,178,36,72]$} & {$[130,76,16,96]$} & {$[169,202,108,240]$} \\
\hline$[3,136,32,64]$ & {$[37,66,88,128]$} & {$[81,242,180,216]$} & {$[133,70,200,240]$} & {$[177,10,148,192]$} \\
\hline$[3,204,144,160]$ & {$[41,18,132,192]$} & {$[83,12,32,128]$} & {$[133,102,72,48]$} & {$[177,18,116,8]$} \\
\hline$[5,22,72,160]$ & {$[41,26,60,128]$} & {$[85,6,96,128]$} & {$[133,166,40,48]$} & {$[177,50,116,72]$} \\
\hline$[5,48,64,128]$ & {$[41,42,204,144]$} & {$[85,66,136,32]$} & {$[133,194,8,48]$} & {$[177,82,132,248]$} \\
\hline$[5,72,208,224]$ & {$[41,50,164,192]$} & {$[85,70,216,32]$} & {$[134,8,80,96]$} & {$[177,162,52,8]$} \\
\hline$[9,10,76,32]$ & {$[41,106,132,112]$} & {$[85,134,216,224]$} & {$[137,10,76,96]$} & {$[177,162,148,88]$} \\
\hline$[9,18,4,32]$ & {$[41,138,228,144]$} & {$[89,18,28,160]$} & {$[137,34,156,192]$} & {$[177,178,228,200]$} \\
\hline$[9,42,4,16]$ & {$[42,36,176,64]$} & {$[89,202,156,32]$} & {$[137,106,4,240]$} & {$[177,194,4,88]$} \\
\hline$[9,66,220,96]$ & {$[49,18,148,248]$} & {$[97,10,60,128]$} & {$[137,130,156,224]$} & {$[177,242,148,248]$} \\
\hline$[9,90,28,32]$ & {$[49,22,184,64]$} & {$[97,34,36,184]$} & {$[137,132,144,224]$} & {$[185,2,172,192]$} \\
\hline$[9,90,36,128]$ & {$[49,34,68,88]$} & {$[97,34,196,72]$} & {$[137,138,44,64]$} & {$[185,146,164,64]$} \\
\hline$[9,130,36,16]$ & {$[49,50,100,232]$} & {$[97,50,100,232]$} & {$[137,154,204,32]$} & {$[193,2,68,56]$} \\
\hline$[9,130,148,64]$ & {$[49,98,164,136]$} & {$[97,66,100,232]$} & {$[137,218,148,224]$} & {$[193,2,104,48]$} \\
\hline$[9,206,80,32]$ & {$[49,114,20,216]$} & {$[97,70,104,16]$} & {$[137,226,36,48]$} & {$[193,6,208,96]$} \\
\hline$[9,218,12,96]$ & {$[49,132,152,64]$} & {$[97,74,172,48]$} & {$[141,202,144,224]$} & {$[193,10,220,96]$} \\
\hline$[10,156,32,64]$ & {$[49,146,52,8]$} & {$[97,130,196,184]$} & {$[145,2,212,232]$} & {$[193,74,212,224]$} \\
\hline$[11,68,80,160]$ & {$[49,146,148,8]$} & {$[97,146,132,72]$} & {$[145,18,4,168]$} & {$[193,98,132,48]$} \\
\hline$[13,42,144,64]$ & {$[49,162,84,24]$} & {$[97,162,20,24]$} & {$[145,82,244,88]$} & {$[193,130,104,176]$} \\
\hline$[13,142,16,64]$ & {$[49,162,180,88]$} & {$[97,162,140,208]$} & {$[145,86,88,96]$} & {$[193,134,72,160]$} \\
\hline$[17,2,84,24]$ & {$[51,132,152,64]$} & {$[97,166,232,112]$} & {$[145,98,68,72]$} & {$[193,146,212,160]$} \\
\hline$[17,2,132,72]$ & {$[65,2,4,88]$} & {$[97,226,148,24]$} & {$[145,98,116,232]$} & {$[193,178,100,136]$} \\
\hline$[17,34,4,168]$ & {$[65,4,8,144]$} & {$[97,226,180,88]$} & {$[145,98,212,184]$} & {$[193,178,116,200]$} \\
\hline$[17,34,84,56]$ & {$[65,10,100,112]$} & {$[97,234,140,112]$} & {$[145,114,212,136]$} & {$[193,194,148,104]$} \\
\hline$[17,66,132,160]$ & {$[65,10,164,112]$} & {$[99,84,40,128]$} & {$[145,130,196,24]$} & {$[193,202,100,240]$} \\
\hline$[17,98,52,200]$ & {$[65,18,244,248]$} & {$[99,100,56,128]$} & {$[145,170,12,64]$} & {$[193,226,12,80]$} \\
\hline$[17,130,156,224]$ & {$[65,66,204,16]$} & {$[101,34,232,16]$} & {$[145,194,164,120]$} & {$[193,234,100,240]$} \\
\hline$[17,134,184,192]$ & {$[65,74,204,208]$} & {$[101,38,8,144]$} & {$[145,202,84,32]$} & {$[194,68,16,32]$} \\
\hline
\end{tabular}

$194,164,104,240]$ $194,164,136,48]$ $195,148,216,224]$ $[197,66,80,96]$ $[197,70,136,176$ $[197,230,232,48$ $[201,14,208,160]$ $[201,138,132,160]$ $[201,202,108,208$ $[203,132,144,96$ $[209,18,196,184$ $[209,18,228,72]$ $209,34,132,200$ $209,66,148,120$ 209, 98, 36, 216] $[209,146,20,24]$ $[209,150,216,32]$ $[209,194,4,24]$ $[209,210,68,136]$ $[209,226,100,216]$ $[211,68,136,32]$ $[213,22,136,32]$ $[213,70,136,160]$ $[217,218,20,160]$ $[225,2,204,144]$ $[225,2,204,144]$ $[225,18,100,136]$ $[225,18,180,232]$ $[225,34,36,152]$ $[225,36,72,80]$ $225,66,100,56]$ $225,66,104,144$ $225,98,196,216$ $[225,130,8,48]$ $[225,130,36,80]$ $[225,130,116,200]$ $[225,166,72,48]$ $[225,194,196,120]$ $[225,198,232,48]$ $[225,202,132,112]$ $[225,226,72,176]$ $[225,242,212,216]$ $[225,242,212,216]$ $[227,100,40,176]$ $[229,102,40,112]$ $[229,102,200,80]$ $229,130,168,240]$ $229,130,200,208]$ $[233,66,172,80]$ $[233,130,100,144]$ $[233,162,12,176]$ $[241,18,132,88]$ $[241,66,4,56]$ $[241,146,148,232]$ $[241,194,84,24]$ $[241,194,180,248]$ $[241,194,180,248]$ 
Table 3: 2 - $(8,4,217 ; 2)$ design $\mathcal{B}_{3}$

\begin{tabular}{|c|c|c|}
\hline$[1,2,4,96]$ & {$[18,8,96,128]$} & {$[65,98,164,232]$} \\
\hline$[1,2,76,176]$ & {$[21,6,136,32]$} & {$[65,98,228,120]$} \\
\hline$[1,2,96,128]$ & {$[25,66,148,96]$} & {$[65,130,228,24]$} \\
\hline$[1,10,32,64]$ & {$[25,74,20,32]$} & {$[65,130,244,72]$} \\
\hline$[1,34,20,136]$ & {$[33,2,72,80]$} & {$[65,134,144,224]$} \\
\hline$[1,34,100,240]$ & {$[33,2,76,112]$} & {$[65,146,68,40]$} \\
\hline$[1,66,164,184]$ & {$[33,2,176,64]$} & {$[65,146,196,56]$} \\
\hline$[1,98,212,136]$ & {$[33,2,212,24]$} & {$[65,154,212,32]$} \\
\hline$[1,106,108,144]$ & {$[33,10,76,128]$} & {$[65,166,104,80]$} \\
\hline$[1,114,196,248]$ & {$[33,10,76,176]$} & {$[65,178,116,216]$} \\
\hline$[1,130,108,48]$ & {$[33,10,100,128]$} & {$[65,202,12,176]$} \\
\hline$[1,136,160,192]$ & {$[33,18,4,24]$} & {$[65,226,148,200]$} \\
\hline$[1,138,68,144]$ & {$[33,100,104,144]$} & {$[65,234,68,112]$} \\
\hline$[1,138,140,240]$ & {$[33,132,8,112]$} & {$[67,36,72,128]$} \\
\hline$[1,146,180,72]$ & {$[33,162,4,216]$} & {$[67,68,72,208]$} \\
\hline$[1,162,12,192]$ & {$[33,194,12,144]$} & {$[67,76,16,32]$} \\
\hline$[1,162,132,56]$ & {$[33,210,52,232]$} & {$[67,132,8,208]$} \\
\hline$[1,194,20,24]$ & {$[33,210,84,200]$} & {$[67,228,136,16]$} \\
\hline$[1,228,136,16]$ & {$[33,226,236,112]$} & {$[69,2,88,128]$} \\
\hline$[1,234,236,16]$ & {$[33,230,72,112]$} & {$[69,14,208,224]$} \\
\hline$[2,8,32,64]$ & {$[33,230,200,112]$} & {$[69,38,232,48]$} \\
\hline$[2,68,40,128]$ & {$[34,8,144,192]$} & {$[69,82,88,32]$} \\
\hline$[2,100,136,240]$ & {$[34,36,168,48]$} & {$[69,102,232,48]$} \\
\hline$[2,148,72,96]$ & {$[34,44,16,64]$} & {$[69,146,152,96]$} \\
\hline$[2,228,72,144]$ & {$[34,132,200,144]$} & {$[69,162,136,80]$} \\
\hline$[2,228,136,112]$ & {$[35,36,184,192]$} & {$[69,214,72,96]$} \\
\hline$[3,116,88,128]$ & {$[35,100,168,48]$} & {$[73,2,4,80]$} \\
\hline$[5,74,48,128]$ & {$[37,34,40,144]$} & {$[73,2,16,224]$} \\
\hline$[5,162,56,64]$ & {$[37,70,136,80]$} & {$[73,10,12,160]$} \\
\hline$[5,162,104,176]$ & {$[37,98,40,128]$} & {$[73,42,172,16]$} \\
\hline$[5,166,200,240]$ & {$[37,198,72,176]$} & {$[73,44,112,128]$} \\
\hline$[9,2,196,208]$ & {$[41,42,132,64]$} & {$[73,74,16,128]$} \\
\hline$[9,6,96,128]$ & {$[41,50,188,192]$} & {$[73,74,148,224]$} \\
\hline$[9,10,12,240]$ & {$[41,66,4,144]$} & {$[73,106,44,208]$} \\
\hline$[9,10,44,48]$ & {$[41,98,84,128]$} & {$[73,122,100,128]$} \\
\hline$[9,10,68,96]$ & {$[41,106,108,16]$} & {$[73,202,220,160]$} \\
\hline$[9,18,156,96]$ & {$[41,106,172,240]$} & {$[73,234,236,48]$} \\
\hline$[9,44,16,128]$ & {$[41,194,196,208]$} & {$[81,38,56,128]$} \\
\hline$[9,76,208,96]$ & {$[49,18,180,8]$} & {$[81,82,148,136]$} \\
\hline$[9,106,4,128]$ & {$[49,26,4,128]$} & {$[81,90,140,96]$} \\
\hline$[9,202,76,176]$ & {$[49,82,116,232]$} & {$[81,98,52,200]$} \\
\hline$[9,202,80,96]$ & {$[49,114,180,88]$} & {$[81,210,116,248]$} \\
\hline$[9,202,148,224]$ & {$[49,178,212,88]$} & {$[81,210,244,8]$} \\
\hline$[11,4,16,64]$ & {$[49,226,164,168]$} & {$[81,226,244,104]$} \\
\hline$[11,12,48,64]$ & {$[49,226,164,232]$} & {$[82,72,32,128]$} \\
\hline$[14,144,160,192]$ & {$[49,242,68,120]$} & {$[82,116,40,128]$} \\
\hline$[17,18,92,160]$ & {$[50,52,72,128]$} & {$[85,150,88,32]$} \\
\hline$[17,26,44,192]$ & {$[51,4,24,128]$} & {$[89,10,204,32]$} \\
\hline$[17,42,60,64]$ & {$[57,58,28,64]$} & {$[89,82,84,32]$} \\
\hline$[17,66,148,232]$ & {$[61,46,64,128]$} & {$[89,106,52,128]$} \\
\hline$[17,114,84,128]$ & {$[65,6,104,80]$} & {$[89,202,4,32]$} \\
\hline$[17,114,164,168]$ & {$[65,10,236,144]$} & {$[89,202,220,224]$} \\
\hline$[17,132,152,160]$ & {$[65,18,156,32]$} & {$[91,12,96,128]$} \\
\hline$[17,134,72,160]$ & {$[65,66,8,48]$} & {$[97,34,164,168]$} \\
\hline$[17,148,200,224]$ & {$[65,66,180,8]$} & {$[97,66,12,144]$} \\
\hline$[17,166,136,64]$ & {$[65,68,136,96]$} & {$[97,66,80,128]$} \\
\hline$[17,178,100,168]$ & {$[65,74,172,80]$} & {$[97,66,100,184]$} \\
\hline$[17,178,188,64]$ & {$[65,82,100,8]$} & {$[97,66,104,80]$} \\
\hline$[17,196,152,224]$ & {$[65,98,132,216]$} & {$[97,74,228,112]$} \\
\hline$[17,218,12,224]$ & {$[65,98,148,136]$} & {$[97,106,140,80]$} \\
\hline
\end{tabular}

$\begin{array}{ll}{[97,162,100,80]} & {[137,194,68,48]} \\ {[97,194,236,16]} & {[137,194,140,224]} \\ {[97,226,100,240]} & {[137,202,4,224]} \\ {[97,226,136,80]} & {[137,226,164,240]} \\ {[97,228,40,240]} & {[141,202,208,96]} \\ {[97,228,200,48]} & {[145,26,84,96]} \\ {[98,4,104,112]} & {[145,50,196,184]} \\ {[98,4,136,144]} & {[145,52,168,192]} \\ {[98,4,168,144]} & {[145,114,36,72]} \\ {[98,164,104,176]} & {[145,146,76,96]} \\ {[98,196,8,80]} & {[145,146,180,64]} \\ {[99,132,232,240]} & {[145,162,116,8]} \\ {[101,106,16,128]} & {[145,178,36,40]} \\ {[105,42,204,176]} & {[145,178,52,192]} \\ {[105,170,4,176]} & {[145,226,244,168]} \\ {[105,194,76,176]} & {[145,242,212,168]} \\ {[113,38,40,128]} & {[153,42,60,192]} \\ {[113,50,196,136]} & {[161,2,136,240]} \\ {[113,82,84,72]} & {[161,34,40,80]} \\ {[113,178,132,120]} & {[161,34,136,176]} \\ {[113,178,228,120]} & {[161,36,200,16]} \\ {[113,210,116,152]} & {[161,54,8,64]} \\ {[129,2,4,248]} & {[161,66,100,216]} \\ {[129,6,216,32]} & {[161,74,108,240]} \\ {[129,10,12,192]} & {[161,98,148,248]} \\ {[129,18,132,104]} & {[161,100,72,16]} \\ {[129,38,16,192]} & {[161,178,148,8]} \\ {[129,38,176,192]} & {[161,194,180,232]} \\ {[129,66,68,200]} & {[162,36,72,80]} \\ {[129,82,20,40]} & {[162,44,48,192]} \\ {[129,82,132,160]} & {[163,100,104,176]} \\ {[129,98,68,168]} & {[163,132,104,112]} \\ {[129,130,92,160]} & {[169,34,140,208]} \\ {[129,130,116,40]} & {[169,34,164,208]} \\ {[129,146,20,104]} & {[169,106,228,48]} \\ {[129,146,52,152]} & {[169,154,180,64]} \\ {[129,146,84,72]} & {[169,186,52,64]} \\ {[129,162,196,176]} & {[169,194,140,208]} \\ {[129,164,48,64]} & {[177,6,56,64]} \\ {[129,178,116,152]} & {[177,18,132,136]} \\ {[129,194,232,80]} & {[177,34,52,192]} \\ {[129,212,88,96]} & {[177,42,132,192]} \\ {[129,218,4,32]} & {[177,66,132,152]} \\ {[129,218,92,32]} & {[177,66,228,136]} \\ {[129,242,4,72]} & {[177,114,132,8]} \\ {[130,100,136,48]} & {[177,114,180,24]} \\ {[130,132,200,176]} & {[177,134,24,192]} \\ {[131,36,200,80]} & {[177,146,196,88]} \\ {[133,6,176,64]} & {[177,162,52,136]} \\ {[133,50,56,192]} & {[177,162,140,192]} \\ {[133,134,48,64]} & {[177,194,132,104]} \\ {[133,134,232,144]} & {[177,226,212,216]} \\ {[133,194,200,144]} & {[179,20,8,192]} \\ {[133,206,208,96]} & {[181,22,184,192]} \\ {[137,2,204,96]} & {[181,178,184,192]} \\ {[137,2,236,176]} & {[185,138,172,192]} \\ {[137,18,156,96]} & {[193,38,72,16]} \\ {[137,34,36,16]} & {[193,50,20,88]} \\ {[137,42,4,208]} & {[193,66,140,32]} \\ {[137,162,236,144]} & {[193,68,8,48]} \\ & \end{array}$

$193,82,200,224]$ $193,106,76,144]$ $193,114,196,120]$ $193,114,228,168]$ $193,140,208,96]$ $193,162,168,16$ $193,178,212,56$ $193,194,232,16]$ $193,196,136,224]$ $193,196,152,96$ $193,226,236,16]$ 193, 226, 244, 136] $194,68,104,176]$ $[195,4,80,32]$ $[197,18,24,32]$ $[197,66,136,240]$ $[197,150,24,224]$ $[197,214,152,32]$ $[197,214,152,32$ $[197,230,40,17$ $[201,2,76,224$ $[201,10,4,208]$ $[201,12,144,32]$ $[201,132,144,96]$ $[201,138,208,224]$ $[201,170,140,144]$ $[209,130,152,32]$ $[209,130,164,200]$ $[209,148,72,96]$ $[209,162,244,184]$ $[209,194,8,96]$ $[209,210,212,216]$ $[213,134,24,96]$ $[225,2,136,112]$ $[225,4,232,176]$ $[225,34,4,56]$ $[225,34,12,80]$ $[225,34,12,208]$ $[225,34,148,40]$ $[225,34,164,240]$ $[225,34,164,240]$ $[225,98,180,200]$ $[225,98,180,200]$ $[225,130,164,112]$ $[225,138,172,16]$ $[225,194,4,168]$ $[225,194,84,40]$ $[225,210,100,168]$ $226,132,72,48]$ $[229,134,104,48]$ $[233,106,68,48]$ $[233,106,140,176]$ $[233,106,140,176]$ $[233,106,164,240]$ $[233,170,44,176]$ $[241,82,52,136]$ $[241,114,116,168]$ $[241,114,116,168]$
$[241,210,20,104]$

Table 4: Realizable large sets used in the proof of Theorem 1

\begin{tabular}{cccc}
$i$ & $\mathrm{LS}_{2}[3]\left(t_{1}, i, 5-i\right)$ & $\mathrm{LS}_{2}[3]\left(t_{2}, k-i, v-6-i\right)$ & $t_{1}+t_{2}+1$ \\
\hline$i \equiv 0 \bmod 6$ & $t_{1}=-1$ & $t_{2}=2$ & 2 \\
$i \equiv 1 \bmod 6$ & $t_{1}=0$ & $t_{2}=1$ & 2 \\
$i \equiv 2 \bmod 6$ & $t_{1}=1$ & $t_{2}=0$ & 2 \\
$i \equiv 3,4,5 \bmod 6$ & $t_{1}=2$ & $t_{2}=-1$ & 2
\end{tabular}


Remark 5.1. We would like to mention that the proof of Theorem 1 works for any value of $q$ and $N$, provided that there exist $\operatorname{LS}_{q}[N](2,3,8)$ and $\operatorname{LS}_{q}[N](2,4,8)$. For example, the parameters $\operatorname{LS}_{3}[7](2,3,8)$ and $\mathrm{LS}_{3}[7](2,4,8)$ are admissible, but the realizability is open. If both large sets actually do exist, then we get an infinite series like in Theorem 1 .

Our knowledge for the existence of $\operatorname{LS}_{2}[3](2, k, v)$ is shown in Table 5. A minus sign indicates that the parameters are not admissible, and a question mark that the parameters are admissible, but the realizability is open. All known realizability results are covered by Theorem 11, In these cases we display the parameter $k$ in the table. Because of duality, only the parameter range $3 \leq k \leq v / 2$ is shown. Apart from the already known $\mathrm{LS}_{2}[3](2,3,8)$ (and its dual $\mathrm{LS}_{2}[3](2,5,8)$ ), all these realizability results are new. The smallest open case is given by the admissible parameter set $\operatorname{LS}_{2}[3](2,6,20)$, which is out of reach of our current construction methods.

\section{Acknowledgement}

The authors would like to acknowledge the financial support provided by COST - European Cooperation in Science and Technology. The authors are members of the Action IC1104 Random Network Coding and Designs over GF(q).

\section{References}

[1] S. Ajoodani-Namini. Extending large sets of t-designs. Journal of Combinatorial Theory, Series A, 76(1):139-144, 1996.

[2] Ronald D. Baker. Partitioning the planes of $A G_{2 m}(2)$ into 2-designs. Discrete Mathematics, 15(3):205-211, 1976.

[3] Zsolt Baranyai. On the factorization of the complete uniform hypergraph. In A. Hajnal, R. Rado, and Vera T. Sós, editors, Infinite and finite Sets, number 10 in Colloquia Mathematica Societatis János Bolyai, pages 91-107, Budapest and Amsterdam, 1975. Bolyai János Matematikai Társulat and North-Holland.

[4] Albrecht Beutelspacher. On parallelisms in finite projective spaces. Geometriae Dedicata, 3(1):35-40, 1974.

[5] Michael Braun. Designs over Finite Fields. In ALCOMA'05 - Proceedings of the Conference on Algebraic Combinatorics and Applications, Designs and Codes, April 3-10, 2005, Thurnau, Germany, pages 58-68. Bayreuther Mathematische Schriften $74,2005$.

[6] Michael Braun, Adalbert Kerber, and Reinhard Laue. Systematic Construction of q-Analogs of Designs. Designs, Codes and Cryptography, 34(1):55-70, 2005.

[7] Michael Braun, Michael Kiermaier, Axel Kohnert, and Reinhard Laue. Large sets of subspace designs. arXiv:1411.7181, 2014. 
Table 5: Admissibility and realizability of $\operatorname{LS}_{2}[3](2, k, v)$

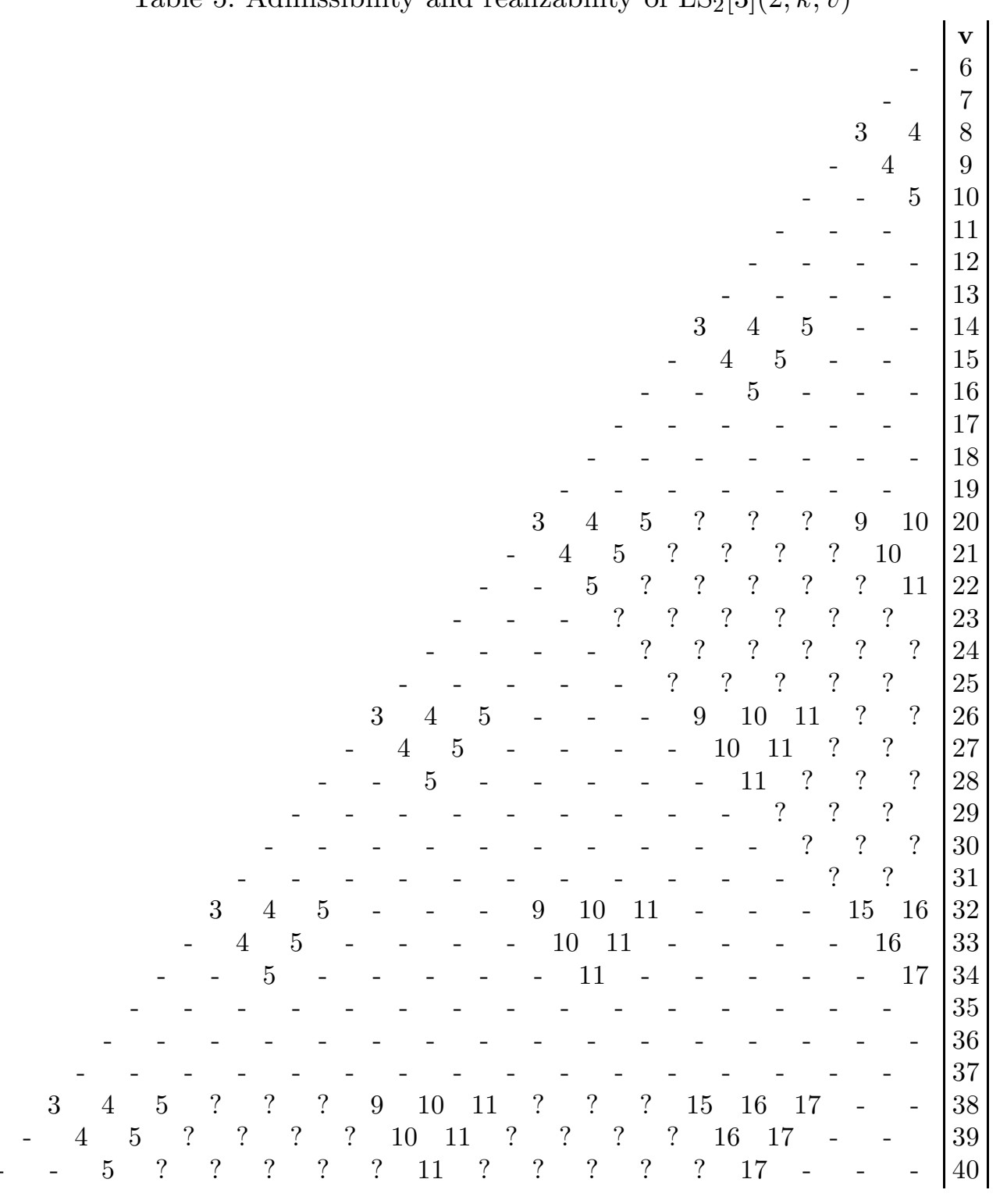


[8] Michael Braun, Axel Kohnert, Patric R. J. Östergård, and Alfred Wassermann. Large sets of t-designs over finite fields. J. Comb. Theory Ser. A, 124:195-202, May 2014.

[9] Peter J. Cameron. Generalisation of Fisher's inequality to fields with more than one element. In Combinatorics, volume 13 of London Mathematical Society Lecture Note Series, pages 9-13. Cambridge University Press, Cambridge, 1974.

[10] Peter J. Cameron. Locally symmetric designs. Geom. Dedicata, 3:65-76, 1974.

[11] Yeow Meng Chee, Charles J. Colbourn, Steven C. Furino, and Donald L. Kreher. Large sets of disjoint $t$-designs. Australasian J. of Combinatorics, 2:111-119, 1990.

[12] Charles J. Colbourn and Jeffrey H. Dinitz. Handbook of Combinatorial Designs, Second Edition (Discrete Mathematics and Its Applications). Chapman \& Hall/CRC, 2006.

[13] Philippe Delsarte. Association Schemes and $t$-Designs in Regular Semilattices. Journal of Combinatorial Theory, Series A, 20(2):230-243, 1976.

[14] Ralph H. F. Denniston. Some packings of projective spaces. Atti della Accademia Nazionale dei Lincei. Rendiconti. Classe di Scienze Fisiche, Matematiche e Naturali. Serie VIII, 52:36-40, 1972.

[15] Tuvi Etzion and Alexander Vardy. Automorphisms of codes in the Grassmann scheme. arXiv:1210.5724, 2012.

[16] Tatsuro Itoh. A New Family of 2-Designs over $G F(q)$ Admitting $S L_{m}\left(q^{l}\right)$. Geometriae Dedicata, 69:261-286, 1998.

[17] G. B. Khosrovshahi and Tayfeh-Rezaie B. Trades and $t$-designs. In Surveys in combinatorics 2009, number 365 in London Mathematical Society Lecture Note Series, pages 91-111. Cambridge Univ. Press, 2009.

[18] Michael Kiermaier and Reinhard Laue. Derived and residual subspace designs. Adv. Math. Commun., 9(1):105-115, 2015.

[19] Earl S. Kramer and Dale M. Mesner. t-designs on hypergraphs. Discrete Math., 15:263-296, 1976.

[20] D. S. Krotov, I. Yu. Mogilnykh, and V. N. Potapov. To the theory of $q$-ary steiner and other-type trades. Discrete Mathematics, 339(3):1150-1157, 2016.

[21] Denis Krotov. The minimum volume of subspace trades. arXiv:1512.02592, 2015.

[22] Masashi Miyakawa, Akihiro Munemasa, and Satoshi Yoshiara. On a Class of Small 2-Designs over $G F(q)$. Journal of Combinatorial Designs, 3:61-77, 1995.

[23] Jumela F. Sarmiento. On point-cyclic resolutions of the 2-(63,7,15) design associated with PG(5,2). Graphs and Combinatorics, 18(3):621-632, 2002. 
[24] Hiroshi Suzuki. 2-Designs over $G F\left(2^{m}\right)$. Graphs and Combinatorics, 6:293-296, 1990.

[25] Hiroshi Suzuki. 2-Designs over GF(q). Graphs and Combinatorics, 8:381-389, 1992.

[26] Simon Thomas. Designs over Finite Fields. Geometriae Dedicata, 24:237-242, 1987.

[27] Ferenc Wettl. On parallelisms of odd dimensional finite projective spaces. Periodica Polytechnica, 19(1-2):111-116, 1991. 